\title{
The Impact of Model-based Therapeutics on Glucose Control in an Intensive Care Unit
}

\author{
Christopher E. Hann ${ }^{1}$, J. Geoffrey Chase ${ }^{1}$, Thomas Desaive ${ }^{2}$, Michael F. Ypma ${ }^{3}$, \\ Jos Elfring ${ }^{3}$ and Geoffrey M. Shaw ${ }^{4}$ \\ ${ }^{1}$ Centre of Bio-Engineering, Department of Mechanical Engineering, University of Canterbury, Christchurch, New Zealand \\ ${ }^{2}$ Institute of Physics, University of Liege, Belgium \\ ${ }^{3}$ Control Systems Technology Group, Department of Mechanical Engineering, Eindhoven University of Technology, Netherlands \\ ${ }^{4}$ Department of Intensive Care, Christchurch Hospital, Christchurch, New Zealand
}

\begin{abstract}
This paper investigates the impact of fast parameter identification methods, which do not require any forward simulations, on model-based glucose control, using retrospective data in the Christchurch Hospital Intensive Care Unit. The integral-based identification method has been previously clinically validated and extensively applied in a number of biomedical applications; and is a crucial element in the presented model-based therapeutics approach. Common non-linear regression and gradient descent approaches are too computationally intense and not suitable for the glucose control applications presented. The main focus in this paper is on better characterizing and understanding the importance of the integral in the formulation and the effect it has on modelbased drug therapy control. As a comparison, a potentially more natural derivative formulation which has the same computation speed advantages is investigated, and is shown to go unstable with respect to modelling error which is always present clinically. The integral method remains robust.
\end{abstract}

Keywords - Glucose control, integral-based parameter identification, model-based therapeutics, Intensive Care Unit

\section{INTRODUCTION}

Therapy guidance using physiological models is a growing trend in bio-engineering [1,2]. Glucose control in the intensive care unit (ICU), has been dramatically improved by using a glucose-insulin model to optimize insulin doses and changes of nutrition [2-4, 9-11]. A glucose control protocol SPRINT (specialized reduced insulin nutrition table) has changed clinical practice in the Christchurch Intensive Care Unit [5]. The result is tight control of blood glucose with a $32 \%$ hospital mortality reduction. Parameter identification is an important part of the overall process, as the identified parameters affect the overall therapy prediction.

An integral-based parameter identification method has been developed [6] and extended to other physiological systems that avoids the need for any forward simulations. It can thus dramatically reduce the computation required. These integral methods are therefore well suited to model- based control applications requiring real-time parameter identification or large Monte Carlo analyses off-line. This paper investigates different computationally fast formulations that don't require forward simulations.

The glucose-insulin model and methods are tested using retrospective clinical data. Several practical issues that arise in clinical implementation are addressed, to highlight issues of performance and stability.

Finally, a new model-based control method for metabolic control is presented, that combines a non-invasive continuous glucose sensor (CGMS) [7] with current standard glucometer sensors [8]. This method is shown to provide a potentially significant improvement in glucose control in simulation that warrants further clinical investigation in the future.

\section{Methodology}

\section{A. Glucose-insulin model}

The glucose-insulin model is defined [6, 9-11]:

$$
\begin{gathered}
\dot{G}=-p_{G} G-S_{I}(t) G \frac{Q}{1+\alpha_{G} Q}+\bar{P} \\
\dot{Q}=-k(Q-I), \dot{I}=-n I+\frac{u}{V}, \bar{P}=P(t)+p_{G} G_{E}
\end{gathered}
$$

where $G(t)$ is the plasma glucose concentration $(\mathrm{mmol} / \mathrm{L})$; $G_{E}$ the equilibrium level of plasma glucose concentration $(\mathrm{mmol} / \mathrm{L}) ; Q(t)$ the interstitial insulin; $I(t)$ the concentration of the plasma insulin above basal level $(\mathrm{mU} / \mathrm{L}) ; P(t)$ the exogenous glucose infusion rate $(\mathrm{mmol} /(\mathrm{L} \mathrm{min})) ; u(t)$ the insulin infusion rate $(\mathrm{mU} / \mathrm{min}) ; V$ the assumed insulin distribution volume (L); $n$ the delay in interstitial transfer of insulin $\left(\mathrm{min}^{-1}\right) ; p_{G}$ the fractional clearance of plasma glucose at basal insulin $\left(\mathrm{min}^{-1}\right) ; S_{I}$ the time-varying insulin sensitivity $(\mathrm{L} / \mathrm{mU} \mathrm{min}) ; k$ the parameter controlling the effective half life of insulin $\left(\mathrm{min}^{-1}\right)$; and $\alpha_{G}$ the MichaelisMenten parameter for glucose clearance saturation. For 
more details on the construction and physiological interpretation of the model Equations (1)-(2) see [6, 9-11].

\section{B. Parameter identification}

For the glucose-insulin Equations (1)-(2), a similar integral-based parameter identification method to [6] is applied. The parameters $\alpha_{G}, k, n$ and $p_{G}$ in Equations (1)(2) are held constant at the population values based on prior studies and sensitivity analysis [6]:

$$
\alpha_{G}=\frac{1}{65}, k=0.0099, n=0.16, p_{G}=0.01
$$

Similarly, the parameter $G_{E}$ is held at the mean glucose of each patient. The carbohydrate input, $P(t)$ in Equations (1) and (2) is also held constant, but may change with respect to time for different patients. The exogenous insulin $u(t)=u_{I}$ is assumed to be a constant infusion over the hour. The parameter $S_{I}$ is insulin sensitivity and is assumed unknown.

Integrating Equation (1) from 0 to $t$ yields and choosing $n$ values of time, $t=t_{1}, \ldots, t_{n}, \in[0,60],\left(0<t_{1}<\cdots<t_{n}\right)$, a set of $n$ equations are formulated:

$G\left(t_{i}\right)-G(0)=-p_{G} \int_{0}^{t_{i}} G d t-S_{I} \int_{0}^{t_{i}} \bar{Q}(t) G(t) d t+\int_{0}^{t_{i}} \bar{P} d t, i=1, \ldots n$

where $\bar{Q}(t)=Q /\left(1+\alpha_{G} Q\right)$. To avoid any error in $\mathrm{G}(0)$ potentially propagating through the equations, $G_{0}=G(0)$ is assumed unknown and is identified along with $S_{I}$. Equations (4) can be written as a matrix system:

$$
\left(\begin{array}{cc}
\int_{0}^{t_{1}} \bar{Q} G d t & -1 \\
\vdots & \vdots \\
\int_{0}^{t_{n}} \bar{Q} G d t & -1
\end{array}\right)\left(\begin{array}{l}
S_{I} \\
G_{0}
\end{array}\right)=\left(\begin{array}{c}
-G\left(t_{1}\right)-p_{G} \int_{0}^{t_{1}} G d t+\int_{0}^{t_{1}} \bar{P} d t \\
\vdots \\
-G\left(t_{n}\right)-p_{G} \int_{0}^{t_{n}} G d t+\int_{0}^{t_{n}} \bar{P} d t
\end{array}\right)
$$

where $G$ is a continuous approximation to the measured glucose [6] and the integrals are evaluated by the trapezium rule. Equation (5) can be solved by linear least squares to determine $S_{I}$ as a constant over any period. Thus, $S_{I}$ may be identified as piecewise constant.

For glucose control in the Intensive Care Unit (ICU), Equation (1) is utilized over periods of 1 hour $[9,11]$ and glucose is measured on the hour. For two glucose measurements $G_{0}=G(0)$ and $G_{60}=G(60)$, the function $G(t)$ in Equation (5) can be approximated by a straight line [6]. For a given infusion $u_{I}$, nutritional input $P(t)$ and glucose measurements $G_{0}$ and $G_{60}$, the solution to Equation (5) determines the required insulin sensitivity. However, note that a similar approach could be used if glucose is measured more frequently.
A similar, potentially simpler, approach to the parameter identification of Equations (4)-(5) is to use the original differential Equations (1)-(2), rather than an integral formulation. For a given set of values, $t=t_{0}, \ldots, t_{n}, n+1$ equations can be formulated:

$$
\dot{G}\left(t_{i}\right)=-p_{G} G\left(t_{i}\right)-S_{I} \bar{Q}\left(t_{i}\right) G\left(t_{i}\right)+\bar{P}, i=0, \ldots, n
$$

where $t_{0}=0$. The analogous matrix system to Equation (5) is defined:

$$
\left(\begin{array}{c}
\bar{Q}\left(t_{0}\right) G\left(t_{0}\right) \\
\vdots \\
\bar{Q}\left(t_{n}\right) G\left(t_{n}\right)
\end{array}\right) S_{I}=\left(\begin{array}{c}
\bar{P}-p_{G} G\left(t_{0}\right)-\dot{G}\left(t_{0}\right) \\
\vdots \\
\bar{P}-p_{G} G\left(t_{n}\right)-\dot{G}\left(t_{n}\right)
\end{array}\right)
$$

where $\dot{G}\left(t_{i}\right)$ are determined by standard finite differences. Equation (7) can be solved by linear least squares to determine $S_{I}$.

This method applies gradients which is similar in concept to typical gradient descent methods. The major difference is that no forward simulations are required so like the integral method [6] it is a computationally fast way of identifying large numbers of $S_{I}$ or other time-varying parameters.

\section{Controlling Drug Delivery}

For the control of blood glucose $G(t)$ in Equation (1), measurements are assumed to be taken every hour with a normally distributed absolute error of $7 \%$, which is typical for a commercial glucometer [8]. Model-based control of glucose typically starts by taking two measurements $G_{0}$ and $G_{60}$ at the times 0 and 60 minutes and computing the insulin sensitivity $S_{I}$ from Equation (5). The goal is to determine the required insulin infusion $u=\mathrm{u}_{\mathrm{I}}$ in Equation (2) that will bring glucose down to a target value $G_{\text {target }}$ in the next hour. This process is performed numerically at each intervention period.

\section{ReSUltS AND DISCUSSION}

\section{A. Glucose control in the Christchurch ICU}

The glucose control protocol SPRINT [3-5] is used extensively in the Christchurch ICU. One of the keys to the success of SPRINT is the significant testing of model-based glucose control algorithms on "virtual" patients prior to implementation. The major physiological variable that is used to represent a "virtual" patient profile is the time varying insulin sensitivity profile in Equation (1) that can be identified from retrospective data. 
The insulin sensitivity profiles provide a means to simulate physiologically realistic time varying glucose response to different insulin and nutrition regimes. This approach thus provides a repeatable cohort for easy comparison of various protocols. It also gives insight into long term clinical performance, and, importantly, lets algorithms and methods be tested safely before clinical implementation.

\section{B. Parameter identification - Integral versus Derivative}

Several numerical studies have been done using data from the retrospective cohort of [6]. For brevity, the results are summarized:

(1) Patients with quite high average insulin infusion, have glucose response close to a straight line between hourly measurements. In this case both the integral and derivative methods perform similarly.

(2) Clinically, periods where the glucose response is significantly different from a straight line can occur when there is an increase in feed from a period of low insulin infusion. During these periods the derivative method greatly underestimates the insulin sensitivity, and in some cases goes negative, even without measurement noise. The result of an underestimated $S_{I}$ value, is an overestimated infusion or bolus which can be dangerous as hyperglycemia may result. This scenario can happen reasonably frequently, so the method of choice is the integral method.

\section{Model-based glucose control}

To demonstrate the practical aspects of model-based glucose control, the integral method is applied to an insulin sensitivity profile from [6]. The patient is Patient 519 , who was a male aged 69; type 2 diabetic; medical subgroup General Surgical; APACHE II score - 29. The nutritional input was initially held constant at $0.049 \mathrm{mmol} /(\mathrm{Lmin}), G_{E}$ was held constant at the mean measured glucose value of $5.84 \mathrm{mmol} / \mathrm{L}$ and the maximum infusion allowed was 6 units.

Fig 1 (a) shows the result of simulated glucose control using the integral method for parameter identification and with $7 \%$ noise placed on the measurements. The mean glucose and standard deviation of $5.58 \pm 1.03 \mathrm{mmol} / \mathrm{L}$ with $67.57 \%$ of glucose values lying in the 4.0 to $6.1 \mathrm{mmol} / \mathrm{L}$ band. The reason for this decrease in performance is that there are significant periods in Figure 14 where the insulin has reached the maximum of 6 Units/hour so effectively no added, but necessary, control is being applied in these periods and insulin effect is saturated $[1,12]$.

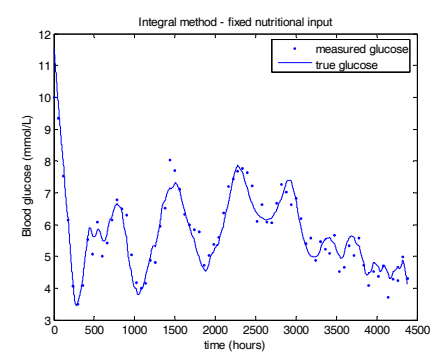

(a)

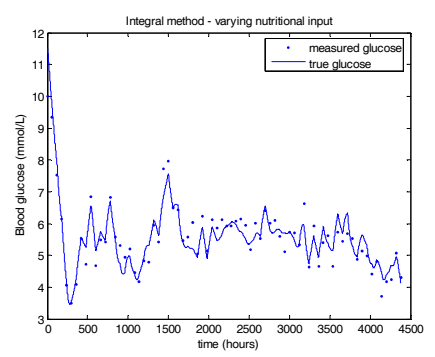

(b)
Fig. 1 (a) Model-based glucose control with the time varying of Figure 12 and a fixed nutritional input given in Equation (22).

(b) Model-based glucose control with the time varying $S_{I}$ and a simply varying nutritional input.

The solution to this problem has been to vary the nutrition, as well as the insulin $[10,11]$. A fully developed and validated method for modulating both the nutrition and insulin in a model-based glycemic control system is detailed in [11].

To demonstrate the essential concept the nutrition is dropped to $40 \%$ of the original value, whenever the insulin hits the upper limit of 6 units. This simple rule results in a significant improvement in glucose control as shown in Fig 1 (b). The mean glucose is $5.32 \pm 0.67 \mathrm{mmol} / \mathrm{L}$ with $76.14 \%$ of values lying between 4 and $6.1 \mathrm{mmol} / \mathrm{L}$.

\section{Combining CGMS with glucocard measurements}

To demonstrate a new clinical application of the methods presented and to further investigate the comparison of the integral versus derivative approaches, a CGMS sensor is included in the model-based glucose control algorithm. The CGMS sensor measures glucose every 5 minutes with a measurement error that can be approximated by the formula:

$$
G_{\text {noise }}=(1+0.18 \delta) G_{\text {true }}, \quad(\mu=0, \sigma=1)
$$

where $\delta$ is the normal distribution. Equation (8) gives a mean absolute error of $14 \%$, which is typical for CGMS sensors [7]. Blood glucose is still assumed to be measured hourly with a glucocard and 7\% uniformly distributed noise in addition to the CGMS for comparison. To account for the extra noise in the CGMS and to give the greatest chance for an averaging effect on the errors, insulin sensitivity $S_{I}$ is fitted over the prior $1 \frac{1 / 2}{2}$ hours rather than 1 hour. The intervention period is also shortened to $1 / 2$ hour to take advantage of the extra measurements from CGMS. The 1 1/2 hour periods ensure that 2 glucocard measurements will always be available to fit $S_{I}$ when stepping along each interval of $1 / 2$ hour. 
A further change that is made is that $7 \%$ low frequency modelling error is added to the glucose measurements, as well as the normally distributed error in Equation (8). The final expression for noise is thus defined:

$$
G_{\text {noise }}=(1+0.18 \delta)\left(1-0.07 \cos \left(\frac{2 \pi}{82} t\right)\right) G_{\text {true }}
$$

Equation (9) reflects the fact that a higher resolution in measurements, trades off with both a higher amount of sensor error and importantly, modelling error.

Fig 2(a) shows the resulting glucose control for Patient 519 using the same parameters as used for Fig 1. A significant improvement can be seen with a mean glucose of $5.03 \pm 0.42 \mathrm{mmol} / \mathrm{L}$ and $98.55 \%$ of glucose values lying between 4 and $6.1 \mathrm{mmol} / \mathrm{L}$.

However, for the derivative method, even with smoothing to remove most of the local noise, a significantly worse result is seen in Fig 2(b). The mean glucose is $5.5 \pm 1.1 \mathrm{mmol} / \mathrm{L}$ with only $64.86 \%$ of glucose values lying between 4.0 and $6.1 \mathrm{mmol} / \mathrm{L}$. Thus, the derivative method is unable to take advantage of the extra CGMS data, where the integral method gives significantly better outcomes on glucose control despite the larger noise distribution for these sensors.

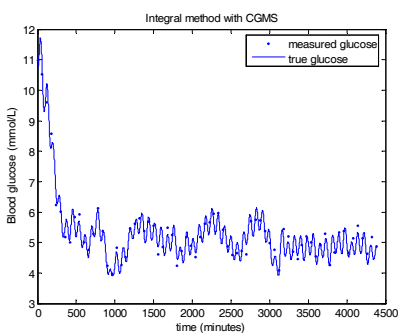

(a)

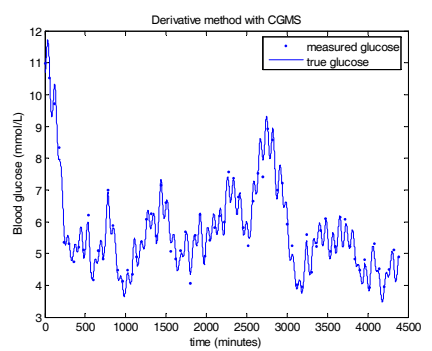

(b)
Fig. 2 (a) Model-based glucose control using the integral method with the combination of a CGMS sensor and glucocard.

(b) Model-based glucose control using the derivative method and with a CGMS sensor and glucocard.

\section{Conclusions}

The main results are summarized:

- The integral formulation in parameter identification is very important for robust and reliable results, particularly with respect to modelling error which is always present in clinical applications.

- The derivative method is very sensitive to modelling error and only works in situations where model response is close to a straight line.
- The combination of the integral method and modelbased drug control is very effective for designing and testing new protocols.

The integral method is thus an important research tool in the model-based therapeutics approach. For example the addition of simulated CGMS shows that a potentially significant clinical gain could be achieved with this continuous sensor. However, further investigation with real CGMS data is required to validate these results. The derivative method, went unstable and failed to realize this possible clinical gain, further emphasizing the importance of integrals in the formulation.

\section{REFERENCES}

1. J. G. Chase, G. M. Shaw, X. W. Wong, T. Lotz, J. Lin, and C. E. Hann, "Model-based Glycaemic Control in Critical Care - A review of the state of the possible," Biomedical Signal Processing and Control, vol. 1, pp. 3-21, 2006.

2. M. Vogelzang, F. Zijlstra, and M. W. Nijsten, "Design and implementation of GRIP: a computerized glucose control system at a surgical intensive care unit," BMC Med Inform Decis Mak, vol. 5, pp. 10-pages, 2005.

3. T. Lonergan, A. L. Compte, M. Willacy, J. G. Chase, G. M. Shaw, C. E. Hann, T. Lotz, J. Lin, and X. W. Wong, "A pilot study of the SPRINT protocol for tight glycemic control in critically Ill patients," Diabetes Technol Ther, vol. 8, pp. 449-62, Aug 2006.

4. T. Lonergan, A. LeCompte, M. Willacy, J. G. Chase, G. M. Shaw, X. W. Wong, T. Lotz, J. Lin, and C. E. Hann, "A Simple InsulinNutrition Protocol for Tight Glycemic Control in Critical Illness: Development and Protocol Comparison," Diabetes Technol Ther, vol. 8, pp. 191-206, 2006.

5. J. G. Chase, G. M. Shaw, A. LeCompte, T. Lonergan, M. Willacy, X. W. Wong, J. Lin, T. Lotz, D. S. Lee, and C. E. Hann, "Implementation and Evaluation of the SPRINT Protocol for Tight Glycaemic Control in Critically Ill Patients: A Clinical Practice Change," Critical Care, vol. 12, p. R49, 2008.

6. Hann CE, Chase JG, Lin J, et al. Integral-based parameter identification for long-term dynamic verification of a glucose-insulin system model. Comput Methods Programs Biomed. 2005 Mar;77(3):259-70.

7. Goldberg PA, Siegel MD, Russell RR, et al. Experience with the continuous glucose monitoring system in a medical intensive care unit. Diabetes Technol Ther. 2004 Jun;6(3):339-47.

8. Arkray. Glucocard ${ }^{\mathrm{TM}}$ Test Strip 2 Data Sheet. Japan: Arkray Inc.; 2001.

9. Chase JG, Shaw GM, Lin J, et al. Adaptive bolus-based targeted glucose regulation of hyperglycaemia in critical care. Med Eng Phys. 2005 Jan;27(1):1-11.

10. Wong XW, Chase JG, Shaw GM, et al. Model predictive glycaemic regulation in critical illness using insulin and nutrition input: a pilot study. Med Eng Phys. 2006 Sep;28(7):665-81.

11. Wong XW, Singh-Levett I, Hollingsworth LJ, et al. A novel, modelbased insulin and nutrition delivery controller for glycemic regulation in critically ill patients. Diabetes Technol Ther. 2006 Apr;8(2): 174-90.

12. Chase JG, Shaw GM, Lin J, et al. Impact of Insulin-Stimulated Glucose Removal Saturation on Dynamic Modelling and Control of Hyperglycaemia. International Journal of Intelligent Systems Technologies and Applications (IJISTA). 2004;1(1/2):79-94. 